\title{
Effects of processing variables and full fat soy flour on nutritional and sensory properties of spaghetti using a mixture design approach.
}

\begin{abstract}
The influence of full-fat soy flour (FFSF) and extrusion conditions on nutritional and sensory characteristics of spaghetti was evaluated using a mixture design, in order to produce functional pasta. Addition of FFSF increases the nutritional composition significantly $(\mathrm{P}<\mathrm{or}=0.05)$. Generally, the presence of FFSF showed a negative influence on the color attribute, but no effect on the firmness and surface condition of spaghetti. No significant difference was observed in the beany flavor up to $23.0 \mathrm{~g} / 100 \mathrm{~g}$. The temperature and screw speed of the extruder had no significant effect on nutritional and sensory attributes, but both enhanced the effect of formulation on the color and surface condition of spaghetti. Also, interaction between the screw speed and the ingredients resulted in a slight positive effect on the firmness and beany flavor attributes. Optimum nutritional value and sensory attributes of spaghetti were produced when $17.0 \mathrm{~g} / 100 \mathrm{~g}$ FFSF was added and processed at a screw speed of $40 \mathrm{rpm}$ and a temperature of 70 degrees $\mathrm{C}$.
\end{abstract}

Keyword: Spaghetti; Enrichment; Extrusion condition; Mixture design; Functional food. 